\title{
Quinones are growth factors for the human gut microbiota
}

\author{
Kathrin Fenn ${ }^{1,3+}$, Philip Strandwitz ${ }^{1 \dagger}$, Eric J. Stewart ${ }^{1}$, Eric Dimise ${ }^{2}$, Sarah Rubin ${ }^{1,4}$, Shreya Gurubacharya', \\ Jon Clardy ${ }^{2}$ and Kim Lewis ${ }^{1 *}$
}

\begin{abstract}
Background: The human gut microbiome has been linked to numerous components of health and disease. However, approximately $25 \%$ of the bacterial species in the gut remain uncultured, which limits our ability to properly understand, and exploit, the human microbiome. Previously, we found that growing environmental bacteria in situ in a diffusion chamber enables growth of uncultured species, suggesting the existence of growth factors in the natural environment not found in traditional cultivation media. One source of growth factors proved to be neighboring bacteria, and by using co-culture, we isolated previously uncultured organisms from the marine environment and identified siderophores as a major class of bacterial growth factors. Here, we employ similar co-culture techniques to grow bacteria from the human gut microbiome and identify novel growth factors.

Results: By testing dependence of slow-growing colonies on faster-growing neighboring bacteria in a co-culture assay, eight taxonomically diverse pairs of bacteria were identified, in which an "induced" isolate formed a gradient of growth around a cultivatable "helper." This set included two novel species Faecalibacterium sp. KLE1255-belonging to the anti-inflammatory Faecalibacterium genus - and Sutterella sp. KLE1607. While multiple helper strains were identified, Escherichia coli was also capable of promoting growth of all induced isolates. Screening a knockout library of E. coli showed that a menaquinone biosynthesis pathway was required for growth induction of Faecalibacterium sp. KLE1255 and other induced isolates. Purified menaquinones induced growth of 7/8 of the isolated strains, quinone specificity profiles for individual bacteria were identified, and genome analysis suggests an incomplete menaquinone biosynthetic capability yet the presence of anaerobic terminal reductases in the induced strains, indicating an ability to respire anaerobically.

Conclusions: Our data show that menaquinones are a major class of growth factors for bacteria from the human gut microbiome. These organisms are taxonomically diverse, including members of the genus Faecalibacterium, Bacteroides, Bilophila, Gordonibacter, and Sutterella. This suggests that loss of quinone biosynthesis happened independently in many lineages of the human microbiota. Quinones can be used to improve existing bacterial growth media or modulate the human gut microbiota by encouraging the growth of important symbionts, such as Faecalibacterium species.
\end{abstract}

Keywords: Uncultured bacteria, Microbiome, Growth factors, Menaquinones, Cultivation, Faecalibacterium

\section{Background}

Research on the microbiome is radically changing our understanding of health and disease [1]. The gut microbiome seems to play an important role in numerous gastrointestinal and metabolic disorders, such as obesity, heart disease, type II diabetes, and cancer [2-5], and

\footnotetext{
*Correspondence: k.lewis@neu.edu; k.lewis@northeastern.edu

${ }^{\dagger}$ Equal contributors

${ }^{1}$ Antimicrobial Discovery Center, Department of Biology, Northeastern University, 134 Mugar Hall, 360 Huntington Ave, Boston, MA 02115, USA Full list of author information is available at the end of the article
}

unexpectedly, the microbiome has also been linked to the nervous system and mental health disorders [6-8].

Traditionally, such microbe-disease-association studies relied heavily on cultivation, yet with the advance of metagenomics, researchers can bypass cultivation to search for links between microbial signatures and disease phenotypes [9]. This is beneficial, as many bacterial species are difficult to grow or have not yet been cultivated. However, any associations between microbiota signatures and health or disease predicted by metagenomics must be confirmed for causality using in vivo 
models, which requires cultivation. Cultivation efforts have improved recovery of microbiome diversity [10, 11], but according to a recent study, 23\% of the 1525 bacterial species identified as residents of the human gut remain uncultured [12].

Previously, we found that growing bacteria in situ in a diffusion chamber enabled growth of uncultured bacteria $[13,14]$. This suggested the existence of growth factors in the natural environment not found in traditional cultivation media. One possible source of such growth factors for uncultured bacteria is other microorganisms. By using co-culture, we found that iron-chelating siderophores produced by neighboring species were growth factors for many uncultured marine bacteria [15]. In the present study, we explored whether a similar phenomenon occurs in the human gut. Here, we report that electron-shuttling quinones, produced by cultivable bacteria, serve as required or growth-promoting factors for a variety of microorganisms of the gut microbiota.

\section{Results}

A screen for uncultured bacteria

We reasoned that some of the colonies that grow on nutrient agar densely plated with a human stool sample are previously uncultured bacteria, only forming because they are in close proximity to a neighboring species producing a growth factor. To explore this, we spreadplated diluted human stool to form $\sim 200$ colonies on a Petri plate seeded with rich agar. Bacteria that depend on growth factors from neighbors will only appear after colonies of the helper species have begun to grow. We therefore noted the slow-growing colonies (the candidate "dependent;" formation observed in 3-7 days), and screened for their dependency on faster growing neighbors (the candidate "helper;" formation observed in 12 days and $<5 \mathrm{~mm}$ away from a candidate dependent). For this, both colonies were resuspended in separate tubes, the late-forming colony was spread-plated on a fresh agar plate, and the faster growing neighbor was then spotted on the same plate (Fig. 1a). We considered a candidate dependent a positive hit if it exhibited enhanced growth around the spotted helper. Apart from this generalized approach, we also tested the ability of Escherichia coli to support growth of neighboring gut bacteria. An inoculum of E. coli K12 BW25113 was spotted on media spread-plated with diluted human stool, and colonies growing near the E. coli spot were then picked and screened for E. coli dependence (Fig. 1b). If growth induction was observed with $E$. coli, a growth factor could then be readily identified by screening a knockout library of E. coli mutants, as we showed previously for siderophores [15].

Using these methods, several "helper-dependent" pairs were identified. After restreaking both the candidate helpers and dependents (with a nearby spot of the helper to stimulate growth) to ensure purity, $16 \mathrm{~S}$ rRNA gene sequencing and annotation showed that the helperdependent bacteria were taxonomically diverse, with two dependents being of low $16 \mathrm{~S}$ rRNA gene sequence similarity to existing type strains (Fig. 2a). In total, after screening roughly 200 candidate helper-dependent pairs, we isolated eight species induced by helper strains. These are Bacteroides clarus KLE1792, Bacteroides intestinalis KLE1704, Bacteroides cellulosilyticus KLE1257, Bacteroides uniformis KLE1607, Bilophila wadsworthia KLE1795, Gordonibacter pamelaeae KLE1812, Faecalibacterium sp. KLE1255, and Sutterella sp. KLE1602 (Fig. 2a). Interestingly, while multiple helper strains were identified in the generalized screen, all isolates exhibiting dependency were also found to be induced by $E$. coli (examples in Fig. 2b-e). Notably, while some of the dependent organisms exhibited growth only near the spotted helper (like B. wadsworthia KLE1795, Fig. 2b), others could form smaller colonies without the presence of the helper, indicating that they can grow on their own, but at a reduced rate. Consequently, we will refer to all isolates as "induced" from here on.

Of particular interest is the previously uncultured Faecalibacterium sp. KLE1255, which is $97.4 \%$ similar by 16S rRNA gene sequence to Faecalibacterium prausnitzii ATCC $27768^{\mathrm{T}}$. Members of the Faecalibacterium genus are generally considered to be beneficial residents of the human gut microbiome due to their antiinflammatory properties [16], likely through induction of IL-10 [17]. Faecalibacterium is negatively correlated with the onset of inflammatory bowel disease [18], further suggesting an important role in human health. Therefore, we decided to isolate the growth factor of Faecalibacterium sp. KLE1255.

\section{Gene coding for the growth factor}

Siderophores, including enterobactin produced by $E$. coli, are a common growth factor for uncultured marine bacteria [15]. We also found that siderophores can be replaced by soluble Fe(II) present at high levels [15]. We therefore tested Fe(II) for its ability to induce growth of the uncultured gut bacteria we identified. However, iron had no effect on the growth of these isolates, suggesting that it is not the missing growth factor (data not shown). We then sought to identify the genes responsible for production of the growth factor and took advantage of the ordered $E$. coli deletion strain collections. The Keio collection is a set of 3985 knockout strains [19] that can be screened for the absence of induction, and thus lead to identification of the genetic locus responsible for production of the growth factor. To simplify the screen, we used strains with larger deletions [20,21], and assembled a minimal set of 283 strains from small-, medium-, and 

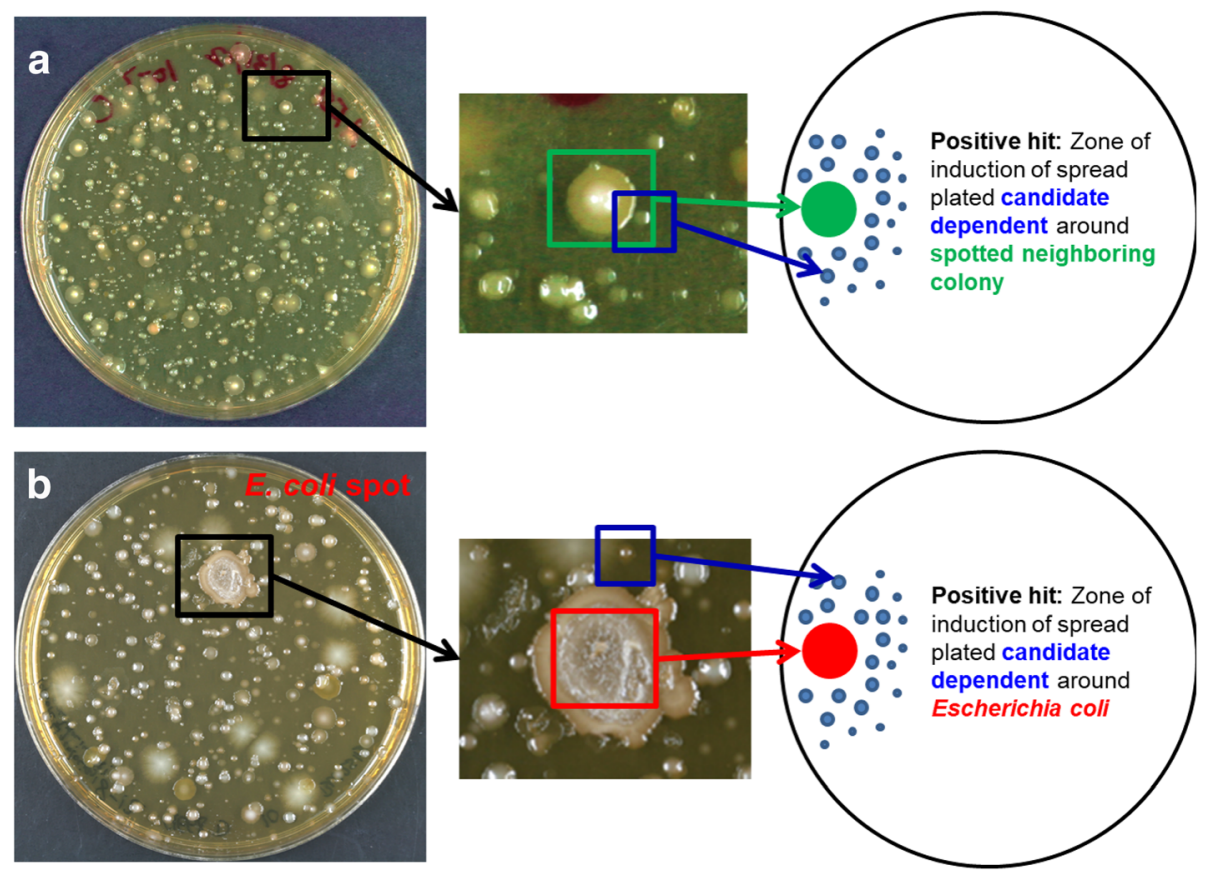

Fig. 1 Isolating uncultured bacteria using co-culture methods. Two approaches are used to identify bacterial isolates from stool exhibiting dependency phenotypes. a In an untargeted approach, early-forming colonies are picked and spotted on plates where late-forming neighboring colonies are spread. $\mathbf{b}$ In a targeted approach, a known helper bacterium, in this case Escherichia coli K12, can be spotted at the time of inoculation of stool sample. Neighboring colonies around the E. coli spot are then picked, spread on fresh agar, and an E. coli inoculum is then spotted on the plate. In both assays, induction of the candidate-dependent bacteria around the spotted helper indicates a positive hit. The plates in this figure are used as a representative example to explain the isolation technique

large-deletion libraries to cover most non-essential genes of the E. coli genome (Additional file 1: Table S1). Strains of this library were then screened individually for induction of Faecalibacterium sp. KLE1255 in co-culture assays, and eight $E$. coli mutants were identified that did not induce growth of Faecalibacterium sp. KLE1255 (Fig. 3a). These mutants harbored deletions in different regions of the genome (Fig. 3b; detail on the missing genes for each strain can be found in Additional file 2: Table S2), but a general theme was an absence of genes

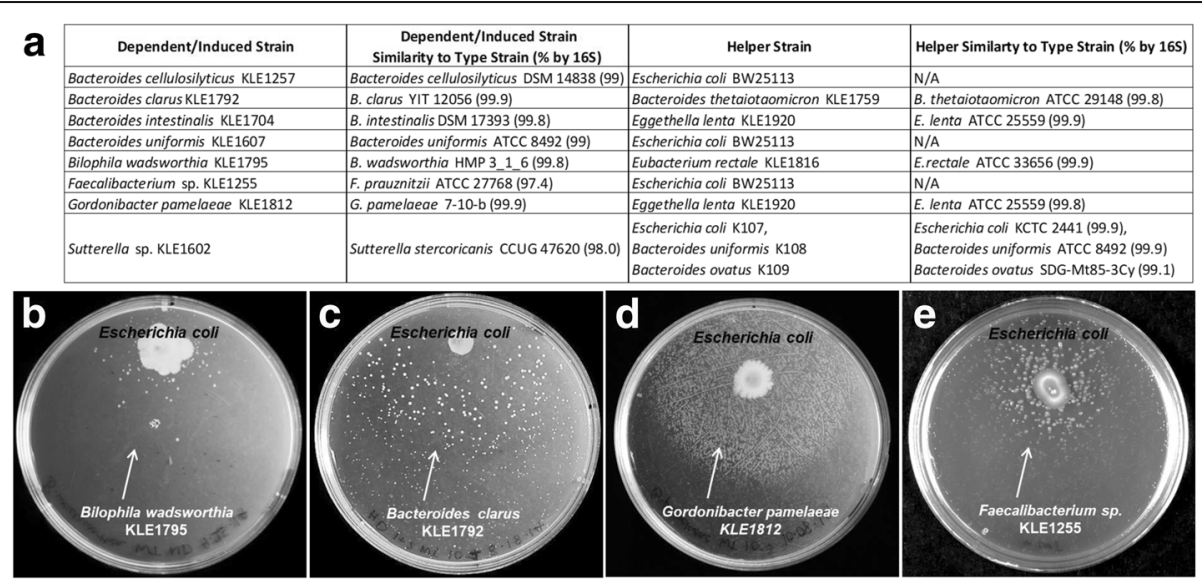

Fig. 2 Escherichia coli is a universal helper for the cultured helper-dependent and helper-induced strains. All strains identified in the co-culture screen were tested for induction by E. coli K12 BW25113. Identified induced strains were spread on brain heart infusion agar with $5.0 \mathrm{~g} / \mathrm{L}$ yeast extract, $0.1 \%$ cysteine, and $15 \mathrm{mg} / \mathrm{mL}$ hemin (BHlych), and 5 L of a 24-h culture of E. coli K12 BW25113 was then spotted on the same plate. a Taxonomic information on the induced isolates and their helper organisms. Different growth induction profiles were identified, including complete dependence, as observed with b Bilophila wadsworthia KLE 1795, and substantially increased colony size, as seen with c Bacteroides clarus KLE1792, d Gordonibacter pamelaeae KLE1812, e Faecalibacterium sp. KLE1255, and all other isolates (not pictured). Images are displayed in black and white to improve contrast 


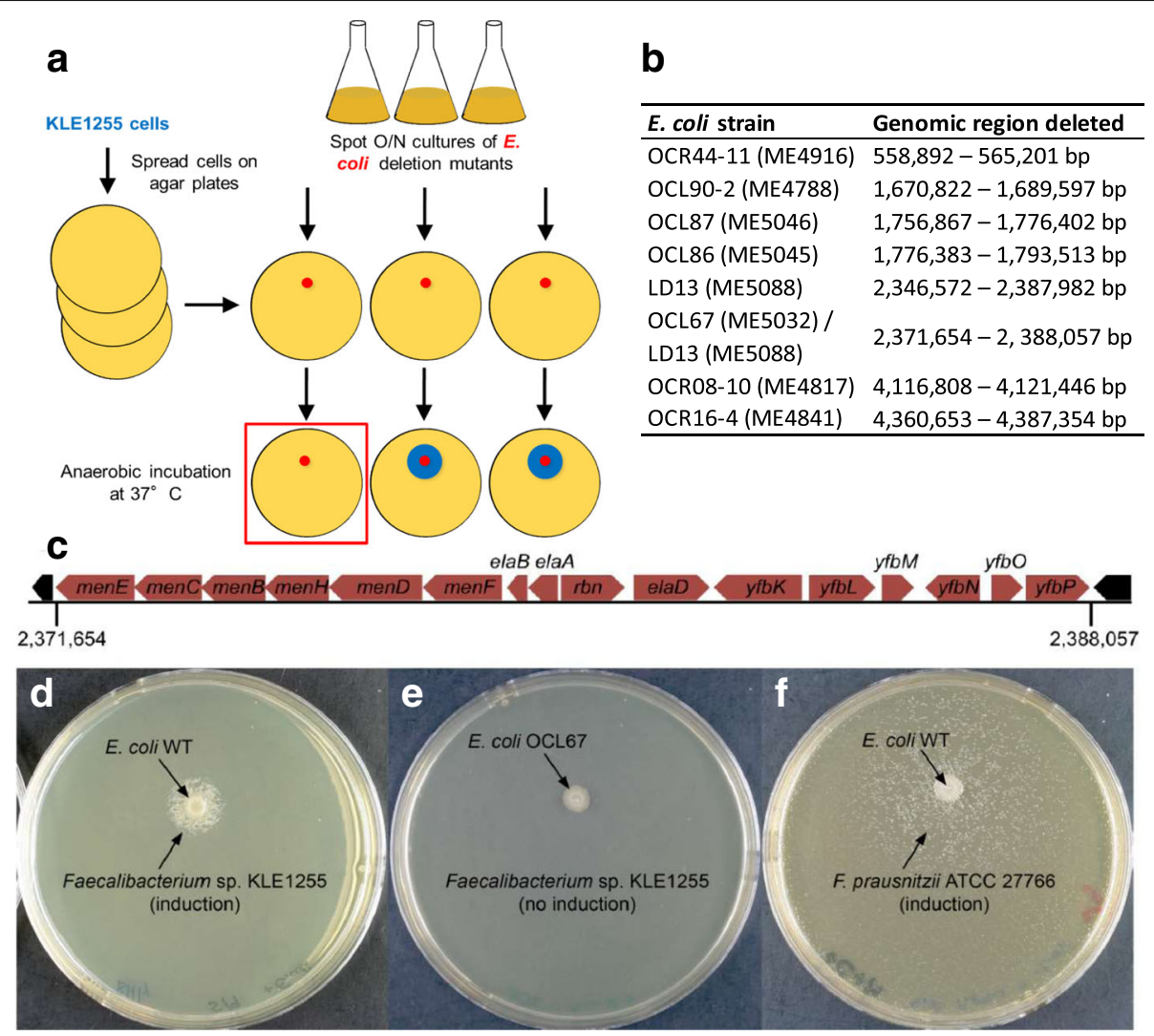

Fig. 3 Screening for deletion mutants unable to induce the growth of Faecalibacterium sp. KLE1255. a A selection of 283 strains that were compiled from E. coli small-, medium-, and large-scale deletion libraries to cover all non-essential genes of the E. coli genome were screened for induction of KLE1 255 via co-culture assay. b Eight strains were identified as being unable to induce growth of KLE1255, harboring deletions in various regions of the genome. $\mathbf{c}$ One strain, E. coli OCL67, had a single large deletion consisting of 16 genes, including 6 in menaquinone biosynthesis. menE = 2-succinylbenzoate-CoA ligase; menC = o-succinylbenzoate synthase; $m e n B=1,4$-dihydroxy-2-naphthoyl-CoA synthase; menH = 2-succinyl-6hydroxy-2,4-cyclohexadiene-1-carboxylate synthase; $m e n D=2$-succinyl-5-enolpyruryl-6-hydroxy-3-cyclohexene-1-carboxylate synthase; men F = isochorismate synthase; $e$ la $B=$ putative membrane-anchored DUF883 family ribosome-binding protein; ela $\mathrm{A}=$ putative N-acetyltransferase; $r b n=$ ribonuclease $\mathrm{BN}$; elaD = deubiquitinase; $y f b K=$ putative lipoprotein; $y f b L=$ putative membrane-associated peptidase; $y f b M=D U F 1877$ family protein; $y f b N=$ uncharacterized protein; yfbO = uncharacterized protein; yfbP = uncharacterized protein. $\mathbf{d}$ Wild type induced strong growth in KLE1255, while e OCL67 did not. Similarly, f WT E. coli also induces Faecalibacterium prausnitzii ATCC 27766

involved with menaquinone biosynthesis. For example, one of the strains unable to induce Faecalibacterium sp. KLE1255, OCL67, has a single 16.4-kb region deleted from its chromosome, which consists of 16 genes, including six genes involved in menaquinone biosynthesis (Fig. 3c, e).

To narrow down the identification of relevant genes, we then tested single gene $E$. coli knockout mutants in the menaquinone, ubiquinone, and chorismate (the precursor to both menaquinone and ubiquinone) biosynthetic pathways from the Keio collection. Strains with deletions in the chorismate and menaquinone biosynthesis pathways were unable to induce growth of Faecalibacterium sp. KLE1255, suggesting that menaquinone was the growth factor for this bacterium (Additional files 3 and 4: Figures S1 and S2). Its cultured relative, F. prausnitzii ATCC 27766, grows on LYHBHI, a BHI medium containing cellobiose and maltose, as well as YCFAG medium. By contrast, Faecalibacterium sp. KLE1255 does not grow on these media. Originally isolated in the presence of rich rumen fluid, F. prausnitzii ATCC 27766 grows poorly, which is a limitation in obtaining sufficient amounts of this symbiont. Consequently, we decided to test the ability of $E$. coli to stimulate growth of $F$. prausnitzii ATCC 27766. Interestingly, in the presence of wild type E. coli, F. prausnitzii ATCC 27766 formed noticeably larger colonies (Fig. 3f).

We similarly tested the other induced isolates identified in the co-culture screen and found that E. coli OCL67, deficient in menaquinone biosynthesis, was unable to stimulate their growth (Fig. 4d). As multiple Bacteroides species were found to be induced by $E$. coli in our original screening efforts, a panel of Bacteroides from our in-house strain collection was also tested for induction by OCL67, to explore whether this was a genus-wide phenomenon. Interestingly, these additional 


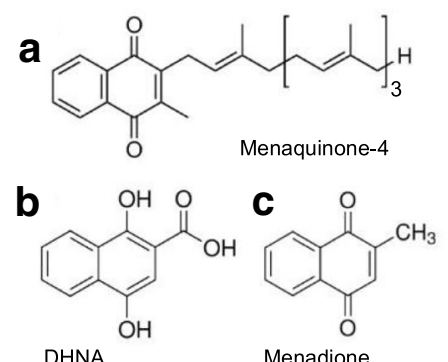

\begin{tabular}{|l|c|c|c|c|c|}
\hline \multirow{2}{*}{ d Strain } & \multicolumn{5}{|c|}{ Induction } \\
\cline { 2 - 6 } & \multicolumn{2}{|c|}{ E. coli } & \multicolumn{3}{|c|}{ Quinones } \\
\cline { 2 - 6 } & WT & OCL67 & DHNA & MK-4 & Men. \\
\hline Faecalibacterium sp. KLE1255 & + & - & - & - & - \\
\hline Bacteroides cellulosilyticus KLE1257 & + & - & + & + & + \\
\hline Bacteroides clarus KLE1792 & + & - & - & + & + \\
\hline Bacteroides intestinalis KLE1704 & + & - & + & - & + \\
\hline Bacteroides uniformis KLE1607 & + & - & + & - & + \\
\hline Bilophila wadsworthia KLE1795 & + & - & + & - & - \\
\hline Gordonibacter pamelaeae KLE1812 & + & - & + & + & - \\
\hline Sutterella sp . KLE1602 & + & - & + & - & - \\
\hline Bacteroides fragilis KLE1758 & - & - & - & - & - \\
\hline Bacteroides thetaiotaomicron KLE1254 & - & - & - & - & - \\
\hline Bacteroides vulgatus K19 & - & - & - & - & - \\
\hline
\end{tabular}
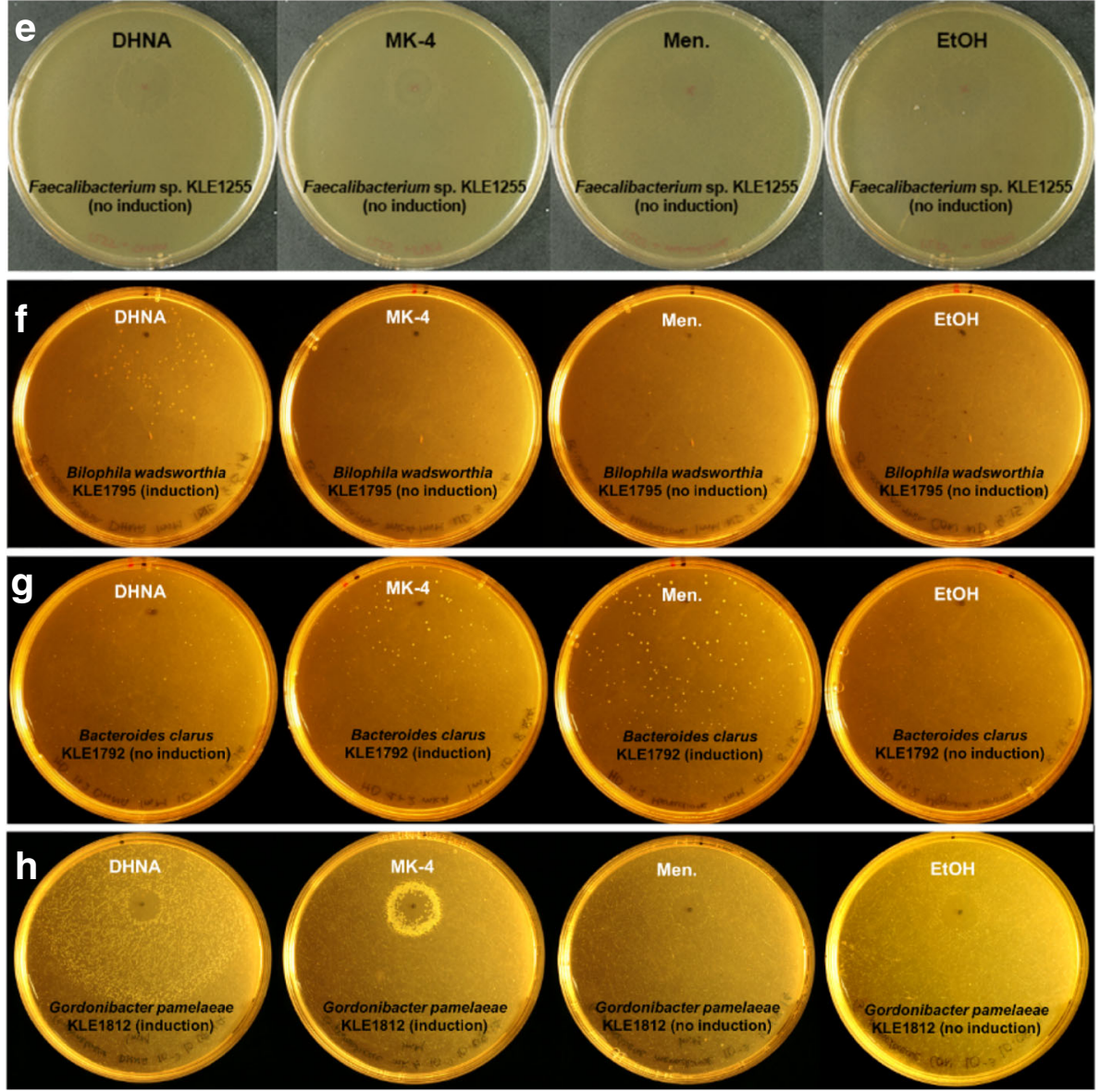

Fig. 4 Quinones are growth factors for E. coli-induced bacteria. Bacteria were plated on rich BHlych agar and spotted with $5 \mu \mathrm{L}$ of $1 \mathrm{mM}$ of a MK-4, b DHNA, or c menadione, or the vehicle of the quinones, ethanol. The exception was KLE1255, which was spotted with $10 \mu \mathrm{L}$ of $10 \mathrm{mM}$ stocks all quinones. $\mathbf{d}$ E. coli and quinone induction capabilities of dependent strains and several cultivable Bacteroides species. Bolded strains represent induced organisms. Examples of induction with purified quinones here include e Faecalibacterium sp. KLE1255, f Bilophila wadsworthia KLE1795, g Bacteroides clarus KLE1792, and h Gordonibacter pamelaeae KLE1812

strains were not induced by $E$. coli but grew perfectly well on their own, suggesting induction can be speciesspecific (Fig. 4d).

\section{Quinones are growth factors for members of the gut microbiota}

To test whether quinones were the growth factor for Faecalibacterium sp. KLE1255 and other isolates induced by $E$. coli, we evaluated three commercially available compounds, menaquinone-4 (MK-4), 1,4-dihydroxy-2- naphthoic acid (DHNA, a biosynthetic precursor of menaquinone), and menadione (a synthetic naphthoquinone ana$\log$ that can act as a menaquinone precursor) (Fig. $4 \mathrm{a}-\mathrm{c}$ ). Suspensions of Faecalibacterium sp. KLE1255 and other organisms induced by E. coli were spread on solid medium, and each quinone was spotted on a separate plate (Fig. $4 \mathrm{~d}-\mathrm{h}$ ). Addition of quinones enabled growth/ induction of seven of the eight isolates tested (Fig. 4d). Interestingly, there was obvious specificity in the ability of quinones to support growth. For example, Blautia 
wadsworthia KLE1795 was induced only by DHNA (Fig. 4b); Bacteroides clarus KLE1792 was induced by MK-4 and menadione, but only weakly by DHNA (Fig. 4d, g); and Gordonibacter pamelaeae KLE1812 was induced by DHNA and MK-4, but not by menadione (Fig. 4d, h). The quinones did not induce growth of the Bacteroides strains we previously found not induced by E. coli (Fig. 4d).

Surprisingly, none of the three commercially available quinones induced the growth of Faecalibacterium sp. KLE1255 (Fig. 4d, e). Considering that this might be due to quinone specificity, as observed with the other strains, we tested additional quinones. However, no induction was observed when KLE1255 was grown in the presence of MK-5, MK-6, MK-7, MK-8, or ubiquinones-Q1, Q2, Q4, Q7, Q8, Q9, and Q10. Menaquinone and ubiquinone are very hydrophobic compounds, and it is possible that $E$. coli is helping with the delivery of these typically insoluble compounds. One possibility is that E. coli delivers its quinones encapsulated in outer membrane vesicles. An attempt to isolate the growth factor of Faecalibacterium sp. KLE1255 from E. coli was not successful, supporting the idea that $E$. coli secretes the quinone in a way to overcome solubility issues.

\section{Genome sequencing of Faecalibacterium sp. KLE1255 reveals a lack of a quinone biosynthetic pathway}

Menaquinone is used in place of ubiquinone in the electron transport chain of some bacteria to transfer electrons during aerobic or anaerobic respiration. To determine the underlying mechanism of menaquinonedependence of Faecalibacterium sp. KLE1255, we sequenced its genome and analyzed it for the presence of various pathways related to electron transport and respiration. KLE1255 does not have gene coding for menaquinone biosynthesis, and this is consistent with reports that no menaquinones could be detected from Faecalibacterium (formerly Fusobacterium) [22, 23]. We did find evidence for NADH dehydrogenase and fumarate reductase in the genome, suggesting that Faecalibacterium sp. KLE1255 possesses a truncated electron transport chain. No other anaerobic reductases were identified in the genome. When comparing the genome of Faecalibacterium sp. KLE1255 to other available Faecalibacterium sp. genomes [24], it became apparent that the components of the respiratory chain identified in these genomes were common among all strains. This may indicate that $F$. prausnitzii has a shortened electron transport chain that may be directed towards fumarate respiration while relying on externally provided menaquinone (Fig. 5).

\section{Discussion}

In this study, we find that diverse members of the human microbiome can only grow (or have substantial growth improvement) in the presence of other gut bacteria. These bacteria come from different generaBacteroides, Faecalibacterium, Bilophila, Gordonibacter, and Sutterella. Different microorganisms, such as Eggerthella lenta, Eubacterium rectale, and several Bacteroides species, serve as "helpers" in these co-culture experiments, but surprisingly, we found that E. coli was able to support the growth of all induced bacteria we isolated. This enabled us to screen a library of $E$. coli gene knockout mutants for the genetic locus responsible for production of the growth factor. Notably, while these kinds of knockout libraries are not readily available for other intestinal bacteria, one could imagine creating and similarly screening an ordered transposon library to identify specific pathways producing growth factors in other helper species.

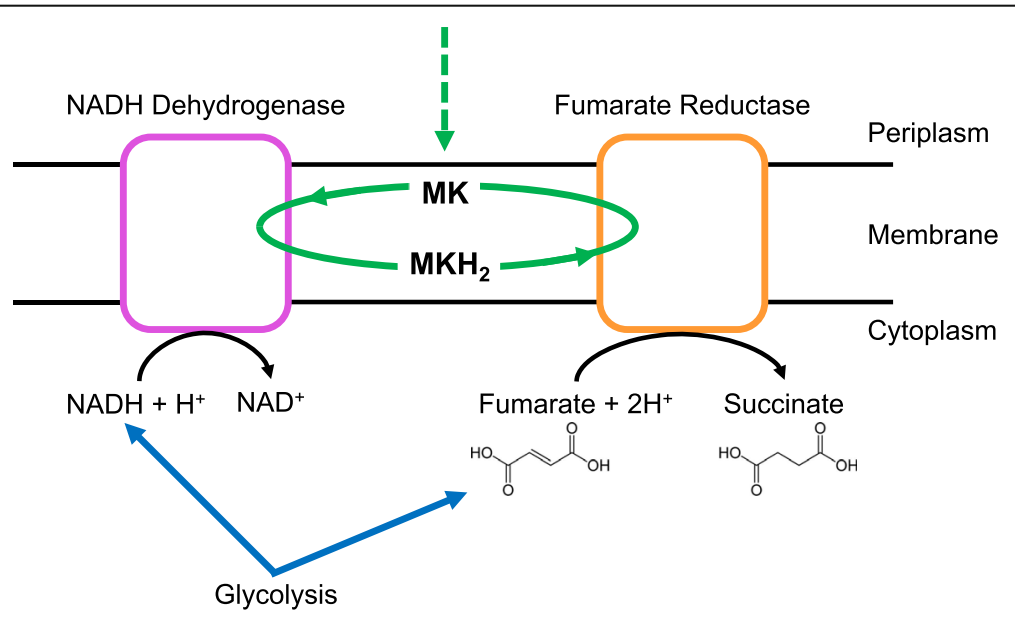

Fig. 5 Model for menaquinone-dependence in Faecalibacterium sp. Genome analysis suggests that Faecalibacterium sp. possess a truncated electron transport chain that is directed towards fumarate respiration. The required menaquinone is provided by an external source (indicated by the dashed arrow). MK menaquinone, $\mathrm{MKH}_{2}$ menaquinol 
Unexpectedly, the screen pointed to a requirement for menaquinone biosynthesis in $E$. coli for it to serve as a universal helper. Menaquinone is a highly hydrophobic component of the anaerobic respiratory chain and would not be expected to serve as an exported growth factor. However, spotting purified menaquinone on a Petri dish produced a ring of growth of several, but not all E. coliinduced isolates. At the same time, a soluble precursor of menaquinone, DHNA, was a better inducer of growth, and superior to menadione, a synthetic precursor of quinones that is present in some growth media and was reported to be an essential growth factor for Prevotella melaninogenicus [25]. P. melaninogenicus does possess the genes $m e n A$ and $u b i E$, which are required to convert DHNA into menaquinone. This suggests that DHNA, secreted by neighboring bacteria, may be the natural growth factor for P. melaninogenicus [25].

Our analysis shows that there is considerable specificity among uncultured bacteria for given quinone compounds, explaining why menadione is not a universal growth factor for these microorganisms. The observed specificity may be driven by variability in the capability to transport menaquinone (or the soluble quinone precursor DHNA); however, transporters for these compounds have not been described to date. Another possible explanation for the specificity profiles could be the capability to convert the menaquinone precursors and synthetic forms into their desired final quinones of choice. In this regard, Faecalibacterium sp. KLE1255 was the only isolate whose growth was not induced by adding purified quinones. Genome sequence of Faecalibacterium sp. KLE1255 showed a complete lack of quinone biosynthesis, but a capability to respire anaerobically. Given Faecalibacterium sp. KLE1255 does not possess the genes required to convert the precursors of menaquinone to a useable form (menA and $u b i E$ ), it likely requires a fully synthesized quinone not tested in our panel.

It is worth mentioning that the original isolation media for Faecalibacterium prausnitzii (formerly Fusobacterium prausnitzii) contained rumen fluid, which likely contains a variety of menaquinones produced by the ruminal microbiota $[26,27]$. Furthermore, it has been reported that $F$. prausnitzii is unable to successfully monocolonize germfree rats, but could when co-colonized with Bacteroides thetaiotaomicron [28] This, coupled with our findings, suggests that members of the Faecalibacterium genus are dependent on quinones from other bacteria to perform anaerobic respiration.

Why some anaerobic bacteria are stringently dependent on respiration is unclear. Indeed, the well-studied E. coli that can respire anaerobically is also capable of growing by fermentation. It is even more puzzling that a bacterium which requires respiration for growth would dispense production of an essential component of the electron transport chain, menaquinone. Our genomic analysis of Faecalibacterium sp. KLE1255 and the identification of other quinone-induced bacteria from the gut microbiota suggests widespread genetic loss of quinone biosynthesis. Recently, 254 genomes of gut bacteria were analyzed for co-occurrence of functional quinone biosynthesis pathways with anaerobic respiratory reductases [24]. One hundred out of 254 of the analyzed genomes had both a complete quinone biosynthetic pathway and terminal respiratory reductases, suggesting widespread respiration potential among the gut microbiota. A large number, nearly $25 \%$, showed a pattern similar to what we found for Faecalibacterium sp. KLE1255-missing or incomplete menaquinone biosynthesis pathways, but the presence of at least one anaerobic respiratory reductase. The species that lost quinone biosynthesis appeared unrelated and included members of the genera Akkermansia, Bacteroides, Bilophila, Clostridium, and Lactobacillus, among others [24].

Notably, closely related type strains of several bacteria we identified in this study had a genetic signature of quinone dependence-an incomplete quinone biosynthesis pathway (Additional file 5: Table S3), yet genes encoding for predicted anaerobic respiratory reductases (Additional file 6: Table S4) [24]. At the same time, other species of the same genera as our quinone-induced isolates did retain the ability to produce their own quinones. For example, the type strain Bacteroides vulgatus ATCC 8482 has both a complete menaquinone biosynthesis pathway and anaerobic reductases (which is supported by the inability of the tested quinones to enhance growth of B. vulgatus K19). In the case of Gordonibacter pamelaeae KLE1812, which we originally isolated in coculture with Eggerthella lenta and showed growth induction by quinones, there are $>20$ anaerobic reductases based on the genome sequence of the type strain (G. pamelaeae DSM 19378). This underscores the need for quinones in bacteria that rely heavily on anaerobic respiration.

Our data suggest that the loss of quinone biosynthesis is an evolutionary recent event and happened independently in different lineages. Apparently, the presence of quinones in the gut allows for the loss of synthesis pathways and the appearance of such "cheaters." The levels of menaquinones produced by $E$. coli or B. vulgatus in monocolonized rats are substantial, 6-8 $\mu \mathrm{g} / \mathrm{g}$ [29], suggesting that these compounds are available to other bacteria. The loss of quinones by gut bacteria is analogous to the loss of siderophores we previously reported for marine uncultured bacteria which obtain them from neighboring species. In both cases, switching from expensive production of an essential component to acquisition will provide growth advantage. This scenario is consistent with the "Black Queen Hypothesis" [30], according to which loss of function at an individual level is favored if it is present among other members of the community. 


\section{Conclusions}

Our data suggest that menaquinones are a major class of required or growth-promoting factors for diverse bacteria from the human gut microbiome. This indicates loss of quinone biosynthesis happened independently in many lineages of the human microbiota. In addition to enabling access to novel diversity from the human microbiome via optimization of culture mediums, there is potential that supplementation of the microbiome with specific quinones or their efficient producers may enrich for desirable symbionts, such as Faecalibacterium species.

\section{Methods}

\section{Human stool collection}

Stool samples from an adult healthy human donor were collected using a commercially available stool collection vessel and with Northeastern University IRB approval. Within 5 min of collection, $1 \mathrm{~g}$ of stool was resuspended in $9 \mathrm{~mL}$ of sterile $20 \%$ glycerol in phosphate-buffered saline (PBS) and homogenized for $30 \mathrm{~s}$ using a vortex. One milliliter aliquots of this mixture was loaded in cryotubes and stored at $-80{ }^{\circ} \mathrm{C}$. For cultivation experiments, individual aliquots of the frozen stool samples were then removed from the freezer and immediately transferred to a Coy Anaerobic Vinyl chamber with an atmosphere of $5 \%$ hydrogen, $10 \% \mathrm{CO}_{2}$, and $85 \%$ nitrogen.

\section{Isolation and cultivation of helper-dependent bacteria}

Serial dilutions of feces samples were spread-plated onto various rich medium (listed below). Plates were then incubated at $37{ }^{\circ} \mathrm{C}$. All experiments were performed under strictly anaerobic conditions. Two different methods were used to obtain helper-dependent organisms: method A. The time of formation for all colonies were tracked for a week. Late-forming colonies (3-7 days) were diluted and spread-plated on fresh medium and nearby $(<5 \mathrm{~mm})$, early-forming colonies (appearance after 1-2 days) were then resuspended in PBS at a high density and spotted $(5 \mu \mathrm{L})$ on the sample plate. The cocultures were incubated for up to 1 week in the chamber and observed daily. Growth induction of the dependent organism around the spotted helper indicated a positive hit. Method B: After spread-plating the feces samples, a helper spot of Escherichia coli K12 BW25113 was spotted on the same plate $(5 \mu \mathrm{L})$. After incubation, colonies were picked in close proximity to the E. coli spot and tested for dependency on $E$. coli in the same way described for method A.

\section{Growth media}

The following growth media were used in this study: brain heart infusion (BHI) supplemented with $5 \mathrm{~g} / \mathrm{L}$ yeast extract, $1 \mathrm{~g} / \mathrm{L}$ cysteine, and $15 \mathrm{mg} / \mathrm{L}$ hemin (only in agar) (BHIych); LYHBHI: BHI supplemented with $5 \mathrm{~g} / \mathrm{L}$ yeast extract, $0.5 \mathrm{~g} / \mathrm{L}$ cysteine, $5 \mathrm{mg} / \mathrm{L}$ hemin, $1 \mathrm{~g} / \mathrm{L}$ cellobiose, $1 \mathrm{~g} / \mathrm{L}$ maltose [16]; fastidious anaerobe agar (FAA); FAA with 5\% $(v / v)$ defibrinated sheep blood added (FAA blood); YCFAG (per liter): $4.5 \mathrm{~g}$ glucose (anhydrous), $10 \mathrm{~g}$ casitone, 2.5 g yeast extract, 4 g $\mathrm{NaCHO}_{3}, 1 \mathrm{~g}$ cysteine, $0.45 \mathrm{~g} \mathrm{~K} \mathrm{~K}_{2} \mathrm{HPO}_{4}, 0.45 \mathrm{~g} \mathrm{KH}_{2} \mathrm{PO}_{4}, 0.9 \mathrm{~g} \mathrm{NaCl}, 0.09 \mathrm{~g}$ $\mathrm{MgSO}_{4} \times 7 \mathrm{H}_{2} \mathrm{O}, 0.09 \mathrm{~g} \mathrm{CaCl}_{2}, 1 \mathrm{mg}$ resazurin, $10 \mathrm{mg}$ hemin, $10 \mu \mathrm{g}$ biotin, $10 \mu \mathrm{g}$ hydroxycobalamin, $30 \mu \mathrm{g}$ paraaminobenzoic acid, $50 \mu \mathrm{g}$ folic acid, $150 \mu \mathrm{g}$ pyridoxamine, $33 \mathrm{mM}$ acetate, $9 \mathrm{mM}$ propionate, $1 \mathrm{mM}$ isobutyrate, $1 \mathrm{mM}$ isovalerate, $1 \mathrm{mM}$ valerate, $50 \mu \mathrm{g}$ thiamine, $50 \mu \mathrm{g}$ riboflavin, $15 \mathrm{~g}$ agar (optional)) [31]. Heat-labile vitamins were added after the medium was autoclaved. All media were prereduced overnight in an anaerobic chamber.

\section{Taxonomic assignment by $16 \mathrm{~S}$ rRNA gene sequencing}

PCR was performed using the general bacterial primers 27F (5'-AGAGTTTGATCMTGGCTCAG-3') and 1492R (5'-TACGGYTACCTTGTTACGACTT-3') to amplify part of the $16 \mathrm{~S}$ rRNA gene. The PCR reaction mixture was $12.5 \mu \mathrm{L}$ GoTaq Master Mix (Promega), $1 \mu \mathrm{L} 10 \mu \mathrm{M}$ 27F and 1492R primers (Operon), $9.5 \mu \mathrm{L}$ Nuclease Free Water (Promega), and $1 \mu \mathrm{L}$ of a colony resuspended in $100 \mu \mathrm{L}$ sterilized distilled water. PCR was performed using an Eppendorf Mastercycler Personal, and the amplification conditions were one cycle of $95^{\circ} \mathrm{C}$ for $5 \mathrm{~min} ; 30$ cycles of $95{ }^{\circ} \mathrm{C}$ for $30 \mathrm{~s}, 55^{\circ} \mathrm{C}$ for $30 \mathrm{~s}, 72^{\circ} \mathrm{C}$ for $90 \mathrm{~s}$; and finally one cycle of $72{ }^{\circ} \mathrm{C}$ for $7 \mathrm{~min}$. Amplification of PCR reactions were confirmed using gel electrophoresis on a $0.8 \%$ agarose gel containing ethidium bromide. Successful PCRs were sequenced by Macrogen Corporation using the 27F primer using the Applied Biosystems 3730xl DNA analyzer. Quality control for sequences was performed using DNA Baser (www.DnaBaser.com), in which ends were trimmed until there were more than $75 \%$ good bases (defined by having a QV score of higher than 25) in an 18-base window. Identification of phylogenetic neighbors and calculations of pairwise sequence similarity were done using the EZTaxon server (http://www.eztaxon.org). $98.7 \%$ similarity by $16 \mathrm{~S}$ was used to determine species level cutoff using a previously suggested level [32].

\section{Screening $E$. coli deletion mutants for a lack of growth induction}

For the library screen, a selection of 283 strains from the E. coli small-, medium-, and large-scale deletion libraries were screened $[20,21]$. The mutants were obtained from the Japanese National BioResource Project. The E. coli strains were grown anaerobically overnight in BHIych. The next day, KLE1255 was plated onto BHIych agar and $1 \mu \mathrm{L}$ spots of $E$. coli cultures were added to the plates. After 2 days of incubation, plates were evaluated for growth of KLE1255. All other induction assays were performed using E. coli suspensions, prepared in 
phosphate-buffered saline or growth medium, rather than overnight cultures as no difference was observed for the different treatments.

\section{Quinone induction experiments}

Inoculums of strains were either created by picking colonies from agar plates seeded with E. coli K12 BW25113 or from 24 to $48 \mathrm{~h}$ cultures in BHIych. These inocula were then bead spread on solid BHIych until dried, and $5 \mu \mathrm{L}$ of a $1-\mathrm{mM}$ stock of DHNA, MK-4, or menadione (all purchased from Sigma and resuspended in 100\% ethanol) was spotted, with ethanol as a control. For Faecalibacterium sp. KLE1255, concentrations of up to $10 \mathrm{mM}$ of DHNA, MK-4, or menadione were also tested. An expanded panel of quinones were also tested for Faecalibacterium sp. KLE1255 induction: MK-8 purified from E. coli K12 BW25113, and MK-4, MK-5, MK-6, MK-7, and MK-8 purified from Micrococcus luteus KLE1011. Ubiquinones tested include Q1, Q2, Q4, Q9, and Q10 (all available from Sigma-Aldrich) as well as Q7 and Q8, which were purified from E. coli. These were tested using multiple solvents (acetone, $\mathrm{MeOH}, \mathrm{DMSO}$, and hexane) at concentrations of $10 \mathrm{mg} / \mathrm{mL}, 1 \mathrm{mg} / \mathrm{mL}$, and $0.1 \mathrm{mg} / \mathrm{mL}$ and $10 \mu \mathrm{L}$ spotted. Methods for isolation of the expanded quinone panel are described below.

\section{Isolation of quinones}

For the expanded quinone panel, quinones were isolated from Escherichia coli K12 BW25113 and Micrococcus luteus KLE1011. Strains were streaked on plates of BHIych or R2Asea, respectively, and individual colonies were picked to inoculate $5.0 \mathrm{~mL}$ cultures, which were grown for $24 \mathrm{~h}$ at $250 \mathrm{rpm}$. These starter preps were used to inoculate $1.0 \mathrm{~L}$ cultures, which were grown for $24 \mathrm{~h}$ at $160 \mathrm{rpm}$. A batch of eight, $1.0 \mathrm{~L}$ cultures was routinely prepared for each organism for cell pellet and supernatant extractions. One liter cultures of bacteria were centrifuged in a Beckman Coulter Avanti J-20 XP centrifuge equipped with a JLA 8.1000 rotor at $4{ }^{\circ} \mathrm{C}$. Cells were pelleted at $4500 \mathrm{rpm}(5053 \times g)$ for $20 \mathrm{~min}$. The supernatant was poured off and the cells were washed with $1 \% \mathrm{NaCl}$. The cells were pooled and pelleted at $6000 \mathrm{rpm}(8983 \times g)$ for $20 \mathrm{~min}$. The supernatant was discarded, and the cells were submitted to quinone extraction. Quinones were isolated either via extraction of saponified cells or via direct solvent extraction of pelleted cells. Saponification was performed in line with established protocols [33]. Briefly, pelleted cells (from $8 \times 1.0 \mathrm{~L}$ preps) were suspended in a $150 \mathrm{~mL}, 3: 2: 1 \mathrm{mix}-$ ture of ethanol:water: $25 \% \mathrm{KOH}$ containing $2.5 \mathrm{~g}$ pyrogallol, transferred to a round bottom flask and refluxed for $20 \mathrm{~min}$ at $100{ }^{\circ} \mathrm{C}$ under an inert atmosphere of dry gas (nitrogen or argon) and under the exclusion of light while stirring vigorously. The mixture was immediately cooled to room temperature in an ice-water bath and then extracted $4 \times$ with equivolume portions of heptane. The organic layers were pooled, dried over anhydrous $\mathrm{Na}_{2} \mathrm{SO}_{4}$, filtered, and concentrated to dryness in vacuo. The dried, oily material was stored under inert gas (nitrogen or argon) at $-20{ }^{\circ} \mathrm{C}$ until chromatographic purification was performed. Direct solvent extraction was performed by suspending pelleted cells (from $8 \times 1.0 \mathrm{~L}$ preps) in $300 \mathrm{~mL}$ of a 3:1 ethanol:diethyl ether solution, transferring to a round bottom flask and stirring vigorously for $2.5 \mathrm{~h}$ under an atmosphere of dry, inert gas and under the exclusion of light. The suspension was filtered, the cells were rinsed with a portion of diethyl ether, and the filtrate was dried to $25 \%$ its original volume in vacuo. The concentrate was diluted with water and extracted $3 \times$ with $500-\mathrm{mL}$ portions of heptane. The pooled organic layer was then dried and stored as described above. Quinones and menachromenols were purified using an Agilent Technologies 1200 Series High Performance Liquid Chromatography system equipped with G1361A Prep Pumps and a G1315D diode array detector. Samples were prepared in $2.0 \mathrm{~mL}$ of acetonitrile and were purified on a Phenomenex Luna C8(2), $250 \times 21.20 \mathrm{~mm}, 5 \mathrm{~mm}$ reverse phase HPLC column. Material was eluted using an isocratic solvent system consisting solely of HPLC grade acetonitrile at a $10-\mathrm{mL} \mathrm{min}^{-1}$ volume flow rate.

\section{NMR characterization of isolated quinones}

All NMR spectra of bacterially derived compounds were acquired using a $\mathrm{CDCl}_{3}$ susceptibility matched $5-\mathrm{mm}$ Shigemi $^{\ominus}$ NMR tube on a Varian VNMRS $600-\mathrm{MHz}$ NMR spectrometer equipped with a 5-mm HCN AutoX inverse probe. Data was acquired using VnmrJ version $\mathrm{X}$ software and was analyzed using MestReNova version 7.0.0-8331 software. Trace acid was removed from "100\%" $\mathrm{CDCl}_{3}$ solvent (99.96 atom \% D-Aldrich) by passing over a plug of Brockmann grade I activated, basic aluminum oxide (Aldrich) immediately before use. Spectra were referenced to TMS or residual protio solvent.

\section{Isolation of genomic DNA from Faecalibacterium sp. KLE1255 for whole genome sequencing}

A phenol/chloroform prep was performed to obtain genomic DNA. Three milliliter of a turbid culture were pelleted and resuspended in $500-\mu \mathrm{L}$ lysis buffer $(20 \mathrm{mM}$ Tris 7.5; $50 \mathrm{mM}$ EDTA; $100 \mathrm{mM} \mathrm{NaCl})$. Fifty microliter of $20 \mathrm{mg} / \mathrm{mL}$ freshly prepared lysozyme and $100 \mu \mathrm{g} / \mathrm{mL}$ proteinase $\mathrm{K}$ were added and the mixture. After 30-60-min incubation, $60 \mu \mathrm{L} 10 \%(w / v)$ sarkosyl (N-lauroylsarkosine) was added and vortexed. Next, $600 \mu \mathrm{L}$ TE-saturated phenol was added; the contents vigorously vortexed for $15 \mathrm{~s}$ and centrifuged for $5 \mathrm{~min}$ at 13,000 rpm. Then, the aqueous phase was removed to a new tube and $600 \mu \mathrm{L}$ phenol/ chloroform were added, the mixture vortexed and the 
aqueous phase removed to a new tube again. One-tenth volume $(60 \mu \mathrm{L})$ of $3 \mathrm{M} \mathrm{NaOAc}$ was added, mixed, and two volumes $\mathrm{EtOH}$ were added. The tube was inverted until the DNA precipitated (4-6 times). The DNA was centrifuged down for $1 \mathrm{~min}$ and the supernatant removed. One-hudred fifty microliter of $70 \%(v / v) \mathrm{EtOH}$ were added and vortexed, and the DNA centrifuged again. The supernatant was removed and the pellet air-dryed for 10-15 min. Finally, the DNA was resuspended in $100 \mu \mathrm{L}$ elution buffer (10 mM Tris-Cl, ph 8.5).

\section{Whole genome sequencing and annotation of Faecalibacterium sp. KLE1255}

Five microliter of genomic DNA was sent to George Weinstock at The Genome Institute at Washington University, St. Louis, Missouri, for Illumina sequencing. The draft genome, consisting of 119 contigs, was annotated using the RAST (Rapid Annotation using Subsystem Technology) server [34] and the KEGG (Kyoto Encyclopedia of Genes and Genomes) database.

\section{Additional files}

Additional file 1: Table S1. Entire chromosome E. coli deletion library reformatted. 283 strains were first compiled from E. coli small-, medium-, and large-scale deletion libraries to cover all non-essential genes of the $E$. coli genome. Strains were taken from the Keio collection [19] and two larger deletion libraries $[20,21]$. (XLS $36 \mathrm{~kb}$ )

Additional file 2: Table S2. Strains identified in the E. coli knockout screen unable to induce the growth of KLE1255. Includes information on which genes are absent for each clone. (XLSX $18 \mathrm{~kb}$ )

Additional file 3: Figure S1. Single deletions in the E. coli chorismate biosynthesis pathway prevented growth induction of KLE1255. Single deletion mutants for all genes involved in chorismate biosynthesis were tested for induction capabilities of KLE1255. Red boxes indicate E. coli mutants with impaired growth induction capabilities for KLE1255. (PNG $449 \mathrm{~kb}$ )

Additional file 4: Figure S2. Single deletions in the E. coli menaquinone-8 pathway, but not ubiquinone-8 pathway, prevented growth induction of KLE1255. Single deletion mutants for all genes involved in ubiquinone-8 and menaquinone-8 biosynthesis were tested for induction capabilities of KLE1255. Red boxes indicate E. coli mutants with impaired growth induction capabilities for KLE1255. (PNG 364 kb)

Additional file 5: Table S3. Quinone-induced bacteria have a disrupted menaquinone biosynthesis pathway, while related organisms not induced by quinones have a complete pathway. The genomes of the nearest type strains of all $E$. coli- or quinone-induced cultured bacteria were surveyed manually for the presence of a functional menaquinone biosynthesis pathway using a published dataset [24]. All organisms induced by E. coli or quinones in earlier co-culture experiments were missing large components of the menaquinone biosynthesis pathway, while Bacteroides species not induced by E. coli or quinones were predicted to have complete menaquinone biosynthetic capabilities. No strains were found to have predicted copies of genes in the futalosine pathway, an alternative means to generate menaquinone. ubiE/menG: 2-methoxy-6-polyprenyl-1,4-benzoquinol methylase; $\operatorname{menF}=$ Menaquinone-specific isochorismate synthase; menD = 2-succinyl-5-enolpyruvyl-6-hydroxy-3-cyclohexene-1-carboxylic-acid synthase; $m e n H=2$-succinyl-6-hydroxy-2,4-cyclohexadiene-1-carboxylate synthase; men Y = 2-succinyl-5-enolpyruvyl-6-hydroxy-3-cyclohexene-1carboxylate dehydrogenase; $m e n C=0$-succinylbenzoate synthase; menE $=0$-succinylbenzoic acid-CoA ligase; $m e n B=$ Naphthoate synthase menl = 1,4-dihydroxy-2-naphthoyl-CoA hydrolase; $m e n J=1,4$-dihydroxy-2-
naphthoyl-CoA hydrolasein (putative); $m e n A=1,4$-dihydroxy-2-naphthoate polyprenyltransferase; $m q n A=$ Chorismate dehydratase; $m a n E=$ Aminodeoxyfutalosine synthase; $m a n C=$ Cyclic dehypoxanthine futalosine synthase; $m a n D=1,4$-dihydroxy-6-naphthoate synthase; manZ = 1,4-dihydroxy-6-naphthoate synthase (alternative); $m a n X=$ Aminodeoxyfutalosine deaminase; $\operatorname{man} B=$ Futalosine hydrolase (EC 3.2.2.26); $m \operatorname{tn} N=$ Aminodeoxyfutalosine nucleosidase; $m a n L=$ 1,4-dihydroxy-6-naphthoate carboxy-lyase, UbiD-like; manM = 2-heptaprenyl1,4-naphthoquinone methyltransferase; $\mathrm{manP}=$ 1,4-naphthoquinone polyprenyltransferase. Data was taken and modified from Racheev, 2016. (XLSX $10 \mathrm{~kb}$ )

Additional file 6: Table S4. Quinone-dependent and control strains have predicted anaerobic reductases. The genomes of nearest type strains of all E. coli- or quinone-induced cultured bacteria were surveyed manually for the presence of individual annotated anaerobic reductases using a published dataset [35]. All analyzed organisms have the genetic capability to utilize anaerobic reductases for anaerobic respiration. Arx = Arsenate reductase; $C y d=$ Cytochrome bd reductase; Dms = Dimethyl sulfoxide reductase; Dsr = Sulfite reductase; Frd = Fumarate reductase; Nap = Nitrate reductase; $\mathrm{Nar}=$ Nitrate reductase; $\mathrm{Nrf}=$ Nitrite reductase; $\mathrm{Phs}=$ Thiosulfate reductase; $\mathrm{Psr}=$ Polysulfite reductase; Tor = Trimethylamine $\mathrm{N}$-oxide reductase; $\mathrm{Ttr}=$ Tetrathionate reductase; $\mathrm{Ynf}=$ Selenate reductase. Data was taken and modified from Racheev, 2014 [35] and Ravcheev, 2016 [24]. (XLSX 8 kb)

\section{Abbreviations}

BHI: Brain heart infusion; BHIYCH: Brain heart infusion supplemented with $5 \mathrm{~g} / \mathrm{L}$ yeast extract, $1 \mathrm{~g} / \mathrm{L}$ cysteine and $15 \mathrm{mg} / \mathrm{L}$ hemin; CFU: Colony-forming units; DHNA: 1,4-Dihydroxy-2-naphthoic acid; FAA: Fastidious anaerobic agar; LYHBHI: BHI supplemented with $5 \mathrm{~g} / \mathrm{L}$ yeast extract, $0.5 \mathrm{~g} / \mathrm{L}$ cysteine, $5 \mathrm{mg} / \mathrm{L}$ hemin, $1 \mathrm{~g} / \mathrm{L}$ cellobiose, $1 \mathrm{~g} / \mathrm{L}$ maltose; MK\#: Menaquinone; \# = length of the isoprenoid sidechain; PBS: Phosphate-buffered saline; Q\#: Ubiquinone; $\#=$ length of the isoprenoid sidechain

\section{Acknowledgements}

The authors would like to thank Dr. George Weinstock and the Genome Institute of Washington University for genome sequencing of Faecalibacterium sp. KLE1255.

\section{Funding}

This work was supported in part by grants R01HG005824 to KL and R01GM086158 to JC.

\section{Availability of data and materials}

Genomic sequence for Faecalibacterium sp. KLE1255 is available on GenBank under accession number NZ_GL538315.1. All strains isolated in this study will be deposited to a public database such as ATCC, and 165 sequences will be deposited in EBI.

\section{Authors' contributions}

$K F, P S$, ES, and KL planned the study and analyzed the data. KF, PS, and KL wrote the paper. KF, PS, SG, and SR performed the co-culture screening for helper-dependent pairs. KF performed the E. coli gene knockout screen. KF analyzed Faecalibacterium sp. KLE1255 genome. ED isolated the expanded quinone panel. PS, KF, SG, and SR performed the quinone specificity assays. All authors helped edit the manuscript. All authors read and approved the final manuscript.

\section{Ethics approval and consent to participate}

All human samples collected for this study were done with Northeastern University institutional review board (IRB) approval (IRB\# 08-11-16), ensuring proper consent and ethical treatment of the participants and their samples.

\section{Consent for publication}

Not applicable.

\section{Competing interests}

The authors declare that they have no competing interests. 


\section{Publisher's Note}

Springer Nature remains neutral with regard to jurisdictional claims in published maps and institutional affiliations.

\begin{abstract}
Author details
${ }^{1}$ Antimicrobial Discovery Center, Department of Biology, Northeastern University, 134 Mugar Hall, 360 Huntington Ave, Boston, MA 02115, USA. 2Department of Biological Chemistry and Molecular Pharmacology, Harvard Medical School, Boston, MA 02115, USA. ${ }^{3}$ Present address: Department of Immunology and Infectious Diseases, Harvard T.H. Chan School of Public Health, Boston, MA 02115, USA. ${ }^{4}$ Present address: Department of Molecular Genetics, Weizmann Institute of Science, 7610001 Rehovot, Israel.
\end{abstract}

Received: 30 August 2017 Accepted: 4 December 2017

Published online: 20 December 2017

\section{References}

1. Walsh CJ, Guinane CM, O'Toole PW, Cotter PD. Beneficial modulation of the gut microbiota. FEBS Lett. 2014;588:4120-30

2. Backhed $F$, et al. Defining a healthy human gut microbiome: current concepts, future directions, and clinical applications. Cell Host Microbe. 2012;12:611-22.

3. Cenit MC, Matzaraki V, Tigchelaar EF, Zhernakova A. Rapidly expanding knowledge on the role of the gut microbiome in health and disease. Biochim Biophys Acta. 2014;1842:1981-92.

4. Garrett WS. Cancer and the microbiota. Science. 2015;348:80-6.

5. Tilg $\mathrm{H}$, Adolph TE. Influence of the human intestinal microbiome on obesity and metabolic dysfunction. Curr Opin Pediatr. 2015;26:496-501.

6. Dinan TG, Stilling RM, Stanton C, Cryan JF. Collective unconscious: how gut microbes shape human behavior. J Psychiatr Res. 2015;63:1-9.

7. Wang $Y$, Kasper $L H$. The role of microbiome in central nervous system disorders. Brain Behav Immun. 2014;38:1-12.

8. Mayer EA, Knight R, Mazmanian SK, Cryan JF, Tillisch K. Gut microbes and the brain: paradigm shift in neuroscience. Neuroscience. 2014;34:15490-6.

9. Schloss PD, Handelsman J. Metagenomics for studying unculturable microorganisms: cutting the Gordian knot. Genome Biol. 2005;6:229.

10. Browne HP, et al. Culturing of 'unculturable' human microbiota reveals novel taxa and extensive sporulation. Nature. 2016;533:543-6.

11. Lau JT, et al. Capturing the diversity of the human gut microbiota through culture-enriched molecular profiling. Genome Med. 2016;8:72.

12. Lagier JC, et al. Culture of previously uncultured members of the human gut microbiota by culturomics. Nat Microbiol. 2016;1:16203.

13. Kaeberlein T, Lewis K, Epstein SS. Isolating "uncultivable" microorganisms in pure culture in a simulated natural environment. Science. 2002;296:1127-9.

14. Ling $L L$, et al. A new antibiotic kills pathogens without detectable resistance. Nature. 2015:517:455-9.

15. D'Onofrio A, et al. Siderophores from neighboring organisms promote the growth of uncultured bacteria. Chem Biol. 2010;17:254-64.

16. Sokol $\mathrm{H}$, et al. Faecalibacterium prausnitzii is an anti-inflammatory commensal bacterium identified by gut microbiota analysis of Crohn disease patients. Proc Natl Acad Sci U S A. 2008;105:16731-6.

17. Rossi $\mathrm{O}$, et al. Faecalibacterium prausnitzii A2-165 has a high capacity to induce IL-10 in human and murine dendritic cells and modulates T cell responses. Sci Rep. 2016;6:18507.

18. Lopez-Siles M, Duncan SH, Garcia-Gil LJ, Martinez-Medina M. Faecalibacterium prausnitzii: from microbiology to diagnostics and prognostics. ISME J. 2017;11:841-52.

19. Baba T, et al. Construction of Escherichia coli K-12 in-frame, single-gene knockout mutants: the Keio collection. Mol Syst Biol. 2006;2:2006.0008.

20. Hashimoto $\mathbf{M}$, et al. Cell size and nucleoid organization of engineered Escherichia coli cells with a reduced genome. Mol Microbiol. 2005;55:137-49.

21. Kato Jl, Hashimoto M. Construction of consecutive deletions of the Escherichia coli chromosome. Mol Syst Biol. 2007;3

22. Ramotar K, Conly JM, Chubb H, Louie TJ. Production of menaquinones by intestinal anaerobes. J Infect Dis. 1984;150:213-8.

23. Fernandez F, Collins MD. Vitamin-K composition of anaerobic gut bacteria. FEMS Microbiol Lett. 1987:41:175-80.

24. Ravcheev DA, Thiele I. Genomic analysis of the human gut microbiome suggests novel enzymes involved in quinone biosynthesis. Front Microbiol. 2016;7:128.
25. Gibbons RJ, Macdonald JB. Hemin and vitamin $\mathrm{K}$ compounds as required factors for the cultivation of certain strains of Bacteroides melaninogenicus. J Bacteriol. 1960;80:164-70.

26. Leedle JA, Hespell RB. Differential carbohydrate media and anaerobic replica plating techniques in delineating carbohydrate-utilizing subgroups in rumen bacterial populations. Appl Environ Microbiol. 1980;39:709-19.

27. Miyazaki K, Martin JC, Marinsek-Logar R, Flint HJ. Degradation and utilization of xylans by the rumen anaerobe Prevotella bryantii (formerly P. ruminicola subsp. brevis) B(1)4. Anaerobe. 1997;3:373-81.

28. Wrzosek $L$, et al. Bacteroides thetaiotaomicron and Faecalibacterium prausnitzii influence the production of mucus glycans and the development of goblet cells in the colonic epithelium of a gnotobiotic model rodent. BMC Biol. 2013;11:61.

29. Kindberg C, Suttie JW, Uchida K, Hirauchi K, Nakao H. Menaquinone production and utilization in germ-free rats after inoculation with specific organisms. J Nutr. 1987;117:1032-5.

30. Morris JJ, Lenski RE, Zinser ER. The Black Queen Hypothesis: evolution of dependencies through adaptive gene loss. MBio. 2012;3

31. Duncan SH, Hold GL, Harmsen HJ, Stewart CS, Flint HJ. Growth requirements and fermentation products of Fusobacterium prausnitzii, and a proposal to reclassify it as Faecalibacterium prausnitzii gen. nov., comb. nov. Int J Syst Evol Microbiol. 2002:52:2141-6.

32. Yarza $P$, et al. Uniting the classification of cultured and uncultured bacteria and archaea using 16S rRNA gene sequences. Nat Rev Microbiol. 2014;12:635-45.

33. Bishop DH, Pandya KP, King HK. Ubiquinone and vitamin K in bacteria. Biochem J. 1962;83:606-14.

34. Aziz RK, et al. The RAST server: rapid annotations using subsystems technology. BMC Genomics. 2008;9:75.

35. Ravcheev DA, Thiele I. Systematic genomic analysis reveals the complementary aerobic and anaerobic respiration capacities of the human gut microbiota. Front Microbiol. 2014;5:674

\section{Submit your next manuscript to BioMed Central and we will help you at every step:}

- We accept pre-submission inquiries

- Our selector tool helps you to find the most relevant journal

- We provide round the clock customer support

- Convenient online submission

- Thorough peer review

- Inclusion in PubMed and all major indexing services

- Maximum visibility for your research

Submit your manuscript at www.biomedcentral.com/submit
) Biomed Central 